\title{
Editorial: The Effects of Altered Gravity on Physiology
}

\author{
Gilles Clement ${ }^{1 *}$, Richard D. Boyle ${ }^{2}$ and Hanns-Christian Gunga ${ }^{3}$ \\ ${ }^{1}$ Lyon Neuroscience Research Center, Bron, France, ${ }^{2}$ National Aeronautics and Space Administration, Washington, DC, \\ United States, ${ }^{3}$ Institute of Physiology, Charité Medical University of Berlin, Berlin, Germany
}

Keywords: adaptation, microgravity, hypergravity, spaceflight adaptation, extreme environment

\section{Editorial on the Research Topic}

\section{The Effects of Altered Gravity on Physiology}

In physiology, a graded dose-response curve relates the stimulus input to a specific measured output. Space studies in humans and animals have provided only a snapshot into understanding the role of gravity on physiological responses. Fully understanding this relationship, including adaptive mechanisms, will provide the information required to ensure normal physiological function in crew for long-duration space missions (Clément, 2017).

Various methods can be used for generating altered gravity, including orbital flight, parabolic flight, head down/up tilt, body loading/unloading, and centrifugation (Richter et al.). In this Frontiers in Physiology Research Topic, ten papers have used these various methods for studying the effects of altered gravity on sensorimotor, musculoskeletal, cardiopulmonary, and cerebrovascular responses in humans and rodents.

\section{ORBITAL FLIGHT}

OPEN ACCESS

Edited and reviewed by: Rachael D. Seidler, University of Michigan, United States

*Correspondence: Gilles Clement gilles.r.clement@nasa.gov

Specialty section: This article was submitted to Environmental, Aviation and Space Physiology, a section of the journal Frontiers in Physiology

Received: 30 September 2019 Accepted: 08 November 2019 Published: 27 November 2019

Citation: Clement G, Boyle RD and Gunga $\mathrm{H}-\mathrm{C}$ (2019) Editorial: The Effects of Altered

Gravity on Physiology.

Front. Physiol. 10:1447.

doi: 10.3389/fphys.2019.01447
Long stays in weightlessness take a toll on the human body, as the muscles atrophy, bones lose minerals, and a new set of stimuli imposes novel challenges on the vestibular and cardiovascular systems (Gunga et al., 2016). Stationary bike and treadmill were used on Sklylab and Mir stations; however, they were not completely successful in preventing astronauts' muscle and bone loss. A new exercise device, the Interim Resistive Exercise Device (iRED), was flown on the International Space Station to target weight-bearing structures, such as squats to load the spine, hips and legs (Schneider et al., 2003). McNamara et al. analyzed computed tomography scans of 16 crewmembers before and after long-duration spaceflight and found that crewmembers experienced less decrease in the back, abdominal, and paraspinal muscles after using iRED protocols in orbit.

During spaceflight, the vestibular otolith organs no longer adequately sense gravito-inertial accelerations. Animal studies have shown that otolith afferents are initially hypersensitive to tilt after return to Earth (Boyle et al., 2001). Perhaps as a result of this hypersensitivity, astronauts overestimate pitch and roll tilt for 1-2 days immediately after landing (Clément and Wood, 2013). In addition, their performance during manual motion-based simulation tasks is greatly impaired, which is a navigational concern (Moore et al., 2019). Clément et al. showed that adaptive changes in astronauts' vestibular processing during spaceflight impair their ability to manually control tilt following transitions between gravitational environments; however, simple aids, such as vibrotactile feedback, can be used to improve their performance.

These changes in vestibular processing after spaceflight are confirmed by brain imaging study showing functional connectivity modifications between the vestibular cortex, the vestibular nuclei, and the cerebellum in cosmonauts after long-duration spaceflight (Pechenkova et al.). Interestingly, the severity of space motion sickness symptoms experienced by these subjects was 
found to correlate with a post- to pre-flight difference in connectivity between the right and left areas of the vestibular cortex.

\section{PARABOLIC FLIGHT}

The parabolic trajectory of an aircraft exposes the passengers to repeated transitions from increased to reduced gravity (Karmali and Shelhamer, 2008). Ritzmann et al. compared postural adjustments in response to perturbations across gravity levels ranging from 0.25 to $1.75 \mathrm{~g}$ by varying the aircraft's pitch angle during pull-up. Subjects walked on a split treadmill and changes in their leg muscles activity and ankle and knee joint trajectories were recorded as the speed of the belts suddenly changed. Results showed a linear relationship between gravity level and EMG amplitudes and muscle onset latencies after perturbations.

\section{HEAD DOWN/UP TILT}

Body tilt of supine subjects allows for partial mechanical unloading along the subject's longitudinal axis in normal Earth's gravity and simulate cephalad fluid shifts (Pavy-Le Traon et al., 2007). Boschert et al. placed subjects at $-12^{\circ}$ (head down), corresponding to $-0.2 \mathrm{~g}$ linear acceleration (Clément et al.), for 3 days and two nights to determine whether changes in cerebral hemodynamics also affected sleep. Results indicated that jugular vein venous congestion occurred faster and that the quality of sleep was poorer at the greater head tilt angle.

Diaz-Artiles et al. compared cardiopulmonary responses of subjects to various gravitational loads along the body longitudinal axis as they exercised on a cycle ergometer while supine with the head-down or head-up. Angles of tilt were $-6^{\circ}$ (simulating $0 \mathrm{~g}), 9.5^{\circ}$ head up ( $0.16 \mathrm{~g}$ lunar gravity), $22.3^{\circ}$ head up $(0.38 \mathrm{~g}$ Mars gravity), and upright ( $1 \mathrm{~g}$ Earth gravity). Heart rate and respiration tidal volume increased as a function of the gravitational load, whereas respiration rate, and the volume of $\mathrm{O}_{2}$ and $\mathrm{CO}_{2}$ uptake decreased as a function of the gravitational loads.

\section{BODY LOADING/UNLOADING}

In rodents, a $30^{\circ}$ head-down tilt by tail suspension unloads the weight-bearing hindlimbs and creates a cephalad fluid shift and situational stress that are analogs to weightlessness (MoreyHolton and Globus, 2002). Ulanova et al. showed that a 7days hindlimb suspension resulted in soleus muscle atrophy and a decrease in titin (T1), a protein responsible for the passive elasticity of muscle. Administration of L-Arginine, a semi-essential amino acid, decreased the degree of atrophy and

\section{REFERENCES}

Boyle, R. D., Mensinger, A. F., Yoshida, K., Usui, S., Intravaia, A., Tricas, T., et al. (2001). Neural readaptation to $1 \mathrm{G}$ following return from space. J. Neurophysiol. 86, 2118-2122. doi: 10.1152/jn.2001.86.4.2118

Clément, G. (2017). International roadmap for artificial gravity research. NPJ Microgravity 3:29. doi: 10.1038/s41526-0170034-8 prevented the decrease in T1 levels, thus mitigating the effects of gravitational unloading.

Mortreux et al. used a partial weight suspension system that allowed for reduced loading on all four limbs while still permitting quadrupedal locomotion (Wagner et al., 2010). Here, rats were supported for 14 days under Mars-analog suspension (38\% weight bearing) and compared with age-matched $(100 \%$ weight bearing) controls. Their results demonstrated that resveratrol, a polyphenol that is known to preserve bone and muscle mass, treatment during partial unloading significantly preserves muscle function and mitigates muscle atrophy.

Arntz et al. studied the vestibular drive for standing balance in humans when loading the body downward at 1.5 and 2 times their body weight, and when exposing subjects to hypergravity $(1.8 \mathrm{~g})$ during parabolic flights. A stochastic electrical vestibular stimulus $(0-25 \mathrm{~Hz})$ delivered during these tasks evoked a vestibular-error signal and corrective muscles responses. With additional load, the magnitude of the vestibular-evoked muscle responses progressively decreased and plateaued at 1.5 times body weight.

\section{CENTRIFUGATION}

Diaz-Artiles et al. exposed subjects to $1.4 \mathrm{~g}$ at their feet via short-radius centrifugation and measured their cardiovascular responses while exercising on a cycle ergometer. They found that cardiac output, stroke volume, pulse pressure, and heart rate significantly increased with the gravity level. These results confirm that combination of exercise and artificial gravity provide a greater cardiovascular benefit than exercise alone.

\section{AUTHOR CONTRIBUTIONS}

All authors listed have made a substantial, direct and intellectual contribution to the work, and approved it for publication.

\section{FUNDING}

This work was supported by the NASA/NAG2945/Neurolab/STS-95 and NIH/NIDCD P01 DC01837 (to RB).

\section{ACKNOWLEDGMENTS}

We sincerely thank the authors who have contributed to the success of this Research Topic. Their articles demonstrate the growing popularity of the field of gravitational physiology and point to promising countermeasures for future space exploration.

Clément, G., and Wood, S. J. (2013). Motion perception during tilt and translation after spaceflight. Acta Astronaut. 92, 48-52. doi: 10.1016/j.actaastro.2012. 03.011

Gunga, H. C., von Ahlefeld, V. W., Appell, H. J., Werner, A. U., and Hoffmann, U. (2016). The Cardiovascular System in Space. Cham: Springer.

Karmali, F., and Shelhamer, M. (2008). The dynamics of parabolic flight: flight characteristics and passenger percepts. Acta Astronaut. 63, 594-602 doi: 10.1016/j.actaastro.2008.04.009 
Moore, S. T., Dilda, V., Morris, T. R., Yungher, D. A., MacDougall, H. G., and Wood, S. J. (2019). Long-duration spaceflight adversely affects post-landing operator proficiency. Sci. Rep. 9:2677. doi: 10.1038/s41598-019-39058-9

Morey-Holton, E. R., and Globus, R. K. (2002). Hindlimb unloading rodent model: technical aspects. J. Appl. Physiol. (1985) 92, 1367-1377. doi: 10.1152/japplphysiol.00969. 2001

Pavy-Le Traon, A., Heer, M., Narici, M. V., Rittweger, J., and Vernikos, J. (2007). From space to earth: advances in human physiology from 20 years of bed rest studies (1986-2006). Eur. J. Appl. Physiol. 101, 143-194. doi: 10.1007/s00421-0070474-z

Schneider, S., Amonette, W., Blazine, K., Bentley, J., Lee, S., Loehr, J., et al. (2003). Training with the international space station interim resistive exercise device. Med. Sci. Sports Exerc. 35, 1935-1945. doi: 10.1249/01.MSS.0000093611. 88198.08
Wagner, E. B., Granzella, N. P., Saito, H., Newman, D. J., Young, L. R., and Bouxsein, M. L. (2010). Partial weight suspension: a novel murine model for investigating adaptation to reduced musculoskeletal loading. J. Appl. Physiol. (1985) 109, 350-357. doi: 10.1152/japplphysiol.00014.2009

Conflict of Interest: The authors declare that the research was conducted in the absence of any commercial or financial relationships that could be construed as a potential conflict of interest.

Copyright (c) 2019 Clement, Boyle and Gunga. This is an open-access article distributed under the terms of the Creative Commons Attribution License (CC BY). The use, distribution or reproduction in other forums is permitted, provided the original author(s) and the copyright owner(s) are credited and that the original publication in this journal is cited, in accordance with accepted academic practice. No use, distribution or reproduction is permitted which does not comply with these terms. 